\title{
THE DIRICHLET PROBLEM FOR ELLIPTIC EQUATIONS WITH DRIFT TERMS
}

\author{
Carlos E. Kenig and Jill Pipher
}

Abstract

We establish absolute continuity of the elliptic measure associated to certain second order elliptic equations in either divergence or nondivergence form, with drift terms, under minimal smoothness assumptions on the coefficients.

\section{Introduction}

In this paper we prove some results on absolute continuity of the elliptic measure associated to a second order elliptic operator under certain natural, minimal conditions on the coefficients of these operators. Primarily, our operators $L$ are of divergence form; that is, $L=\operatorname{div} a \nabla$ where $a(X)=\left(a_{i j}(X)\right)$ is strongly elliptic in the sense that there exists a positive constant $\lambda$ such that

$$
\lambda|\xi|^{2} \leq \sum_{i, j} a_{i j}(x) \xi_{i} \xi_{j}<\lambda^{-1}|\xi|^{2},
$$

for all $X$ and all $\vec{\xi} \in \mathbb{R}^{n}$. However, thanks to some recent work of S. Hoffman and J. Lewis $[\mathbf{H L}]$, we can extend our results to operators with a drift term, i.e., $L+b \nabla$, under certain conditions on $b$. These conditions on $b$ will, in turn, yield information for non-divergence operators as well. One feature of these theorems is that we need not assume that the matrix $\left(a_{i j}\right)$ is symmetric.

Let us motivate the condition we shall place on the matrix $a$ of $L=$ $\operatorname{div} a \nabla$ by the following example.

Consider the Laplacian $\Delta=\sum_{i} D_{x_{i} x_{i}}$, in a domain $\Omega$ above the graph of a Lipschitz function $\phi$, and let $d \omega$ denote the harmonic measure on the boundary of $\Omega$, with respect to some fixed interior point. In [D1], Dahlberg proved the mutual absolute continuity of $d \omega$ with respect to $d \sigma$, the surface measure on $\partial \Omega$, and showed that the density $k=d \omega / d \sigma$

Partially supported by the NSF. 
satisfies a reverse Hölder condition of order 2. One approach to studying this harmonic measure in domains with Lipschitz boundary is to map $\Omega$ to the upper half space $\mathbb{R}_{+}^{n+1}$, and look at the resulting pull-back of $\Delta$ under this transformation. If one simply "flattens" the domain

$$
\Omega=\left\{(x, t) \in \mathbb{R}^{n} \times \mathbb{R} \mid t \geq \phi(x)\right\}
$$

by the map $(x, t) \mapsto(x, t-\phi(x))$, then the Laplacian is mapped to a symmetric divergence form operator $L=\operatorname{div} a \nabla$ whose coefficients $a=\left(a_{i j}\right)$ are merely bounded and measurable, since they depend on the derivatives of the Lipschitz function $\phi$. In general, the elliptic measures associated to the entire class of divergence structure elliptic operators, with bounded, measurable coefficients, may be singular with respect to the surface measure on the boundary. (See $[\mathbf{C F K}]$ for details.) But the operator $L$ arising from the transformation above possesses additional structure - the coefficients $a_{i j}$ are independent of the $t$ variable. It seemed reasonable to conjecture that this independence in the $t$ variable would imply that the general class of such operators would satisfy the same reverse Hölder estimates that the density for $\Delta$ satisfies on $\partial \Omega$. This was shown in $[\mathbf{J K}]$ and led to a series of works on exactly how to relax the requirement of independence in the $t$ variable. The paper $[\mathbf{F K P}]$ contains some sharp results in this direction, as well as many of the references to previous work.

On the other hand, there is a more useful transformation discovered by B. Dahlberg, C. Kenig and E. Stein ([D2]) mapping $\mathbb{R}_{+}^{n}$ to $\Omega$ of the form

$$
\rho(x, t)=\left(x, c t+\theta_{t} * \phi(x)\right),
$$

where $c$ is a constant that depends on $\|\nabla \phi\|_{\infty}$ and can be chosen large enough to insure that $\rho$ is one-one. The function $\theta \in C_{0}^{\infty}\left(\mathbb{R}^{n}\right)$ is even, and $\theta_{t}(\cdot)=t^{-n} \theta(\cdot / t)$. The pullback of $\Delta$ from $\Omega$ to $\mathbb{R}_{+}^{n+1}$ is also a symmetric elliptic operator, $L=\operatorname{div} a \nabla$, where $a$ possesses the properties:

(i) $|\nabla a(x, t)| \leq C / t$.

(ii) $t|\nabla a(x, t)|^{2} d x d t$ is a Carleson measure.

(For the definition of Carleson measure, see (2.3).)

In 1984, Dahlberg posed two conjectures. The first conjecture concerned perturbation of operators. Suppose that, in the upper half space $\mathbb{R}_{+}^{n+1}$, one has an elliptic operator $L_{0}=\operatorname{div} A_{0} \nabla$ for which the Dirichlet problem $D_{p}$ with data in $L^{p}\left(\mathbb{R}^{n}, d x\right)$ is solvable. Now suppose $L_{1}=$ $\operatorname{div} A_{1} \nabla$ is a perturbation of $L_{0}$ in the sense that $\left|A_{1}(x, t)-A_{0}(x, t)\right|^{2} d x \frac{d t}{t}$ 
is a Carleson measure. Then, is the Dirichlet problem $D_{q}$ for $L_{q}$ also solvable, where $q$ may be larger than $p$ ? (The conjecture can be stated equivalently in terms of the weight condition $d \omega_{L_{1}} \in A_{\infty}\left(d \omega_{L_{0}}\right)$ - see Definition (2.5).) This conjecture was solved affirmatively in $[\mathbf{F K P}]$, where references to earlier work (such as [D2]) may also be found. It is, however, the second conjecture that is the subject of this paper, and which concerns, not perturbations of operators, but classes of operators whose coefficients satisfy conditions (i) and (ii) above. Here the question is whether or not the Dirichlet problem $D_{p}$ for some $p$ is solvable for such an $L$ - equivalently, whether the $A_{\infty}$ condition holds.

Until recently, most positive results proving $A_{\infty}$ estimates for a class of elliptic operators relied on $L^{2}$ identities, in the spirit of [JK], which in turn relied on symmetry assumptions. ([FJK] is one interesting exception to this.) But there are a variety of reasons for studying the non-symmetric situation. These include the connections with nondivergence form equations and the broader issue of obtaining estimates on elliptic measure in the absence of $L^{2}$ identities which relate tangential and normal derivatives.

In $[\mathbf{K K P T}]$, the study of nonsymmetric divergence form operators with bounded measurable coefficients was initiated. In particular, some sharp $A_{\infty}$ estimates were proven for certain operators in the class associated with the first transformation (flattening) mentioned above - that is, the operators on $\mathbb{R}^{2}$ whose coefficients are independent of one of the variables. This result was an application of a new method of establishing mutual absolute continuity, and $A_{\infty}$ ([KKPT, Theorem 2.3]).

In this paper, we show that this same technique can be used to prove $A_{\infty}$ results for elliptic measures of operators satisfying the bounds and the Carleson measure conditions (i) and (ii) above. In the next section, we make some definitions and state our main results. We thank Steve Hofmann for helpful discussions on these topics and on his work [HL].

\section{Some definitions - Statements of the main theorems}

Definition 2.1. $\mathbf{Z} \subset \mathbb{R}^{n}$ is an $M$-cylinder of diameter $d$ if there exists a coordinate system $(x, t)$ such that

$$
\mathbf{Z}=\{(x, t):|x| \leq d,-2 M d \leq t \leq 2 M d\}
$$

and for $s>0$,

$$
s \mathbf{Z}=\{(x, t):|x|<s d,-2 M d \leq t \leq 2 M d\} .
$$


Definition 2.2. $\Omega \subset \mathbb{R}^{n}$ is a Lipschitz domain with Lipschitz 'character' $\left(M, N, C_{0}\right)$ if there exists a positive scale $r_{0}$ and at most $N$ cylinders $\left\{Z_{j}\right\}_{j=1}^{N}$ of diameter $d$, with $\frac{r_{0}}{c_{0}} \leq d \leq c_{0} r_{0}$ such that

(i) $8 Z_{j} \cap \partial \Omega$ is the graph of a Lipschitz function $\phi_{j}$,

$$
\left\|\phi_{j}\right\|_{\infty} \leq M, \phi_{j}(0)=0 \text {. }
$$

(ii)

$$
\partial \Omega=\bigcup_{j}\left(Z_{j} \cap \partial \Omega\right)
$$

(iii)

$$
Z_{j} \cap \Omega \supset\left\{(x, t):|x|<d, \operatorname{dist}((x, t), \partial \Omega) \leq \frac{d}{2}\right\} .
$$

If $Q \in \partial \Omega$ and

$$
B_{r}(Q)=\{x:|x-Q| \leq r\}
$$

then $\Delta_{r}(Q)$ denotes the surface ball $B_{r}(Q) \cap \partial \Omega$ and $T\left(\Delta_{r}\right)=\Omega \cap B_{r}(Q)$ is the Cartesian region above $\Delta_{r}(Q)$.

Definition 2.3. A measure $d \mu$ defined in $\Omega \subset \mathbb{R}^{n}$ is a Carleson measure (denoted $d \mu \in \mathcal{C}$ ) if there exists a constant $C>0$ such that for all $r \leq r_{0}$,

$$
\mu\left(T\left(\Delta_{r}\right)\right) \leq C \sigma\left(\Delta_{r}\right)
$$

where $d \sigma$ is the surface measure of $\partial \Omega$.

Let $\tilde{L}=\operatorname{div} a \nabla+b \cdot \nabla$, where $a=\left(a_{i j}\right)$ and $b=\left(b_{j}\right)$ are matrices with bounded measurable coefficients and $\left(a_{i j}\right)$ satisfies the elliptic condition (1.1). We may assume that $a$ is symmetric without any loss of generality for operators satisfying the hypothesis of Theorem 2.8 (see Remark 2.12). A function $u$ in the Sobolev space $W_{1, \text { loc }}^{2}(\Omega)$ is said to be a weak solution to $L$ in $\Omega$ if

$$
\int_{\Omega} a \nabla u \cdot \nabla \phi-(b \cdot \nabla u) \phi d X=0
$$

for all $\phi \in C_{0}^{\infty}(\Omega)$.

When $b \in L^{\infty}$, weak solutions are in fact Hölder continuous, and satisfy an interior Harnack inequality, by the DiGiorgi-Nash-Moser theory (see $[\mathbf{G T}]$, for example). Our assumptions on $b$ will be weaker, but will imply that $b \in L^{\infty}$ in the interior. 
Associated to $L=\operatorname{div} a \nabla+b \cdot \nabla$, and a domain $\Omega \subset \mathbb{R}^{n}$, is a family of elliptic measures $d \omega_{L}^{X}$ for $X \in \Omega$. These are the representing measures of solutions to $L$ in $\Omega$ which arise from the solvability of the continuous Dirichlet problem: $L u=0$ in $\Omega, u_{\mid \partial D}=g \in C(\partial \Omega)$. The unique solvability of this continuous Dirichlet problem was one of the results of $[\mathbf{H L}]$ for operators like $L$ which have drift terms $b \cdot \nabla$, under certain conditions on $b$. We shall be more specific about this following the statements of Theorems 2.6 and 2.8 below.

Fixing some $X \in \Omega$ we set $d \omega_{L}=d \omega_{L}^{X}$, and refer to $d \omega_{L}$ as the elliptic measure for $L$ in $\Omega$. The solvability of the Dirichlet problems for $L$, when the boundary data belongs to $L^{p}(\partial \Omega, d \sigma)$, for some $p$ and for $d \sigma$ denoting surface measure on $\partial \Omega$, depends upon the precise relationship between $d \omega_{L}$ and $d \sigma$. To help quantify this, we recall some definitions.

Definition 2.5. If $d \mu$ and $d \nu$ are doubling measures on $\partial \Omega$, then $d \mu \in$ $A_{\infty}(d \nu)$ if there exists a constant $c>0$ and $\delta$ such that, for any $\Delta \subset \partial \Omega$ and any set $E \subset \Delta$,

$$
\frac{\mu(E)}{\mu(\Delta)} \leq C\left(\frac{\nu(E)}{\nu(\Delta)}\right)^{\delta}
$$

As the constant in Definition 2.5 is independent of the sets $E$ and $\Delta$, the $A_{\infty}$-condition is in fact a quantitative, or uniform, version of mutual absolute continuity. If $d \omega_{L} \in A_{\infty}(d \sigma)$ then it will follow from the general theory of weights $([\mathbf{C F}],[\mathbf{M}])$ that there exists $p<+\infty$ for which the Dirichlet problem $D_{p}$ for $L$ on $\Omega$ with data in $L^{p}(\partial \Omega, d \sigma)$ is uniquely solvable. (See $[\mathbf{F K P}]$ and the references therein for details.)

The main results of this paper are the following.

Theorem 2.6. Let $L=\operatorname{div} a \nabla$ be an elliptic operator and let $\Omega \subset \mathbb{R}^{n}$ be a bounded Lipschitz domain. Let $\delta(X)=\operatorname{dist}(X, \partial \Omega)$, and suppose that $a=\left(a_{i j}\right)$ has distributional derivatives satisfying

$$
\sup \left\{\delta(X)\left|\nabla a_{i j}(X)\right|^{2}: X \in B_{\delta(Z) / 2}(Z)\right\}
$$

is a Carleson measure in $\Omega$. Then, the elliptic measure $d \omega_{L}$ associated to $L$ belongs to $A_{\infty}(d \sigma)$.

Theorem 2.8. If $\tilde{L}=L+b \cdot \nabla$, where $L$ is as in Theorem 2.6, and

$$
\sup \left\{\delta(X)|\vec{b}(X)|^{2}: X \in B_{\delta(Z) / 2}(Z)\right\}
$$

is a Carleson measure in $\Omega$.

Then $d \omega_{\tilde{L}} \in A_{\infty}(d \sigma)$. 
Remark 2.10. We do not assume, in Theorem 2.6, that the matrix $\left(a_{i j}\right)$ is symmetric. It was observed in [KKPT], that the results of [CFMS] for operators $L=\operatorname{div} A \nabla$ with merely bounded measurable coefficients were valid even when $A$ was not symmetric. Thus, for operators of Theorem 2.6, the existence of elliptic measure, its relationship to the Green's functions and various classical important properties of solutions (Harnack property, Hölder continuity of weak solutions, comparison principles) are all valid (see (1.3)-(1.14) of [KKPT]).

Remark 2.11. The Carleson measure conditions 2.7 and 2.9 of the theorems are stated in terms of supremums so that they imply certain essential pointwise estimates on $a_{i j}$ and $b_{j}$. For example, (2.9) implies that $|\vec{b}(X)| \leq C \delta(X)^{-1}$ for $x \in \Omega$, and (2.10) implies an analogous estimate on $\left|\nabla a_{i j}\right|$.

Remark 2.12. In the statement of Theorem 2.8, the symmetry of the matrix $a=\left(a_{i j}\right)$ is no longer a factor. That is, it suffices to assume that $\left(a_{i j}\right)$ is symmetric, and the general case will follow. To see this, let $L=D_{i}\left(a_{i j}\right) D_{j}+b \cdot \nabla$, and let us assume (2.8) holds for $a=\left(a_{i j}\right)$ symmetric, satisfying (2.7). Consider a non-divergence form operator $L_{0}=$ $a_{i j} D_{i} D_{j}+b \cdot \nabla$ where $\vec{b}$ satisfies (2.9), and where $\left(a_{i j}\right)$ need not be symmetric. Let $A_{i j}=\frac{a_{i j}+a_{j i}}{2}$, so that $L_{0}=A_{i j} D_{i} D_{j}+B \cdot \nabla$. Then $L_{0}$ may also be written as

$$
L_{0}=D_{i}\left(A_{i j}\right) D_{j}+\left(B-\left(D_{i} A_{i j}\right)_{j}\right),
$$

and we see that the resulting lower order term $B-\left(D_{i} A_{i j}\right)_{j}$ also satisfies (2.9). Thus $L_{0}$ satisfies the hypotheses of Theorem 2.8. Now observe that

$$
L=D_{i}\left(a_{i j} D_{j}\right)+b \cdot \nabla u=a_{i j} D_{i} D_{j}+\left(b+D_{i} a_{i j}\right)_{j} \cdot \nabla,
$$

i.e., $L$ is an operator whose structure is the same as $L_{0}$, to which the theorem applies.

The above observations give rise to an interesting corollary of Theorem 2.8, namely that the same conclusion applies to operators in nondivergence form. Moreover, we also see that it now suffices to prove (2.6) assuming $\left(a_{i j}\right)$ is symmetric, although the method of proof does not distinguish between the two cases.

In light of the fact that the matrices $\left(a_{i j}\right)$ for $L$ in the statement of Theorem 2.8 may be assumed symmetric, Theorem 2.8 follows from Theorem 2.6 by applying the perturbation Theorem 2.17 of [HL]. From $A_{\infty}$ for the operator $L$ it will follow that $A_{\infty}$ holds for $\tilde{L}=L+b \cdot \nabla$ under conditions (2.9) on the drift terms. In [HL], the authors prove 
$A_{\infty}$ estimates for parabolic operators as well as elliptic ones, and must therefore deal with the non-trivial issues of the lack of a doubling condition on parabolic measures. But in the elliptic case, their conditions on the coefficients of the matrix $\left(a_{i j}\right)$ are more restrictive than ours, so Theorem 2.6 is not contained in their work. However, the perturbation methods they develop are powerful, and we did not find a simpler means of obtaining (2.8) from (2.6) without invoking their Theorem 2.17.

Theorem 2.6 will follow from establishing the hypotheses of Theorem 2.9 of [KKPT], which gives a Littlewood-Paley criterion for $A_{\infty}$ estimates. To state the theorem, we recall the classical operators associated with the concepts of mathematical convergence of solutions at the boundary, and of uniqueness in the Dirichlet problem. Our domains will be assumed to be Lipschitz, and hence we may define the non-tangential approach regions, for each $Q \in \partial \Omega$, by

$$
\Gamma_{\alpha}(Q)=\{X \in \Omega:|X-Q| \leq(1+\alpha) \operatorname{dist}(X, \partial \Omega)\}
$$

which are compactly contained in $\Omega$ when $\alpha$ is sufficiently large. The parameter $\alpha$ is the aperture of the cone. Sometimes, we shall need to truncate the cones at height $d$ and we denote by $\Gamma_{\alpha, d}(Q)$ this truncated cone $\Gamma_{\alpha}(Q) \cap B_{d}(Q)$. The square function of $u$, defined in $\Omega \subset \mathbb{R}^{n}$, at a point $Q \in \partial \Omega$ relative to a family of cones $\left\{\Gamma_{\alpha, d}\right\}$ is defined by:

$$
S_{\alpha, d} u(Q)=\left\{\int_{\Gamma_{\alpha, d}(Q)}|\nabla u(X)|^{2} \operatorname{dist}(X, \partial \Omega)^{2-n} d X\right\}^{\frac{1}{2}} .
$$

The non-tangential maximal function of $u$ is defined by

$$
N_{\alpha, d} u(Q)=\sup \left\{|u(X)|: X \in \Gamma_{\alpha, d}(Q)\right\}
$$

Definition 2.13. The Dirichlet problem $\left(D_{p}\right)$ with data in $L^{p}(\partial \Omega, d \sigma)$ is solvable for $L$ if whenever $f \in \mathcal{C}(\partial \Omega)$, the solution $u$ to the continuous Dirichlet problem with data $f$ satisfies the estimate:

For fixed $\alpha, d$, there exists $C>0$, depending on $\alpha$ and on ellipticity, such that

$$
\left\|N_{\alpha, d}(u)\right\|_{L^{p}(d \sigma)} \leq C\|f\|_{L^{p}(d \sigma)} .
$$


The nontangential maximal function $N(u)$, when $u_{\mid \partial \Omega}=f$ is comparable to a maximal function of Hardy-Littlewood type relative to the measure $d \omega_{L}$ :

$$
M_{\omega_{L}} f(Q)=\sup _{\Delta \ni Q} \int_{\Delta} f(P) \frac{d \omega_{L}(P)}{\omega_{L}(\Delta)} .
$$

Thus, by the theory of weights, $D_{p}$ is solvable for $L$ if and only if $d \omega_{L}=$ $k d \sigma$ and $k$ satisfies a reverse Hölder inequality of order $p^{\prime}, \frac{1}{p}+\frac{1}{p^{\prime}}=1$. Again, $d \omega_{L} \in A_{\infty}(d \sigma)$ if and only if a reverse Hölder inequality for the density is valid for some (possibly large) choice of exponent, and hence $d \omega_{L}$ belongs to $A_{\infty}(d \sigma)$ if and only if $\left(D_{p}\right)$ is solvable for some $p$. One of the main results of [KKPT] is the following criterion for establishing $A_{\infty}$ for operators $L$ in divergence form.

Theorem 2.15 (2.9 of [KKPT]). Let $L=\operatorname{div} A \nabla$ be elliptic and $A=$ $\left(a_{i j}\right)$ be a matrix of bounded measurable functions. Let $\Omega$ be a bounded Lipschitz domain. Suppose that for all Lipschitz subdomains $D \subset \Omega$ one has the $L^{p}$ norm equivalence, for some $p>0$,

$$
\begin{aligned}
\|N(u)\|_{L^{p}\left(\partial \Omega^{\prime}, d \sigma\right)} & \leq C_{1}\|S(u)\|_{L^{p}\left(\partial \Omega^{\prime}, d \sigma\right)} \\
& \leq C_{2}\|N(u)\|_{L^{p}\left(\partial \Omega^{\prime}, d \sigma\right)}
\end{aligned}
$$

for constants $C_{1}, C_{2}$ depending only on the Lipschitz character of $\Omega$. Then $d \omega_{L} \in A_{\infty}(d \sigma)$ on $\Omega$.

We note that Theorem 2.15 was stated for $p=2$, but once (2.16) holds for some choice of $p$, it holds for all, by a purely real variable argument. And finally, we showed that in [KKPT] that Theorem 2.15 was sharp in the sense that no conclusion stronger than $A_{\infty}$ could be drawn. Theorems 2.6 and 2.8 are sharp as well, in the same sense. In [FKP], a class of divergence form operators (of Beurling-Ahlfors type) was shown to be sharp for the $A_{\infty}$ condition. A routine computation verifies that their coefficients always satisfy the condition (2.7) of Theorem 2.6, and hence the conclusion cannot be improved.

\section{Proof of Theorem 2.6}

The main results of the paper are proven by establishing the hypothesis of Theorem 2.15 above. That is, on every sub-domain $\Omega^{\prime}$ of $\Omega$ we will prove the $L^{p}$ norm equivalence of the square function and the non-tangential maximal function with respect to surface measures on the boundary. The first lemma permits us to assume that the Carleson measure condition (2.7) holds on every Lipschitz subdomain of our original domain. 
For $\Omega \subset \mathbb{R}^{n+1}$, a Lipschitz domain, let

$$
\epsilon_{\Omega}(X)=\sup \left\{|\nabla a(Z)|: Z \in B\left(X, \delta_{\Omega}(X) / 2\right\}\right.
$$

where $\delta_{\Omega}(X)=\operatorname{dist}(X, \partial \Omega)$. If $\Omega=\mathbb{R}_{+}^{n+1}$, we omit the subscript and simply write $\epsilon(x, t)$ where $X=(x, t) \in \mathbb{R}^{n} \times \mathbb{R}_{+}$.

Lemma 3.1. Suppose that $\Omega \subset \mathbb{R}^{n+1}$ is a Lipschitz domain and that $\delta_{\Omega}(X) \epsilon_{\Omega}^{2}(X) d X$ is a Carleson measure with norm $C$. Then, on every bounded Lipschitz subdomain $\Omega^{\prime}$ of $\Omega, \delta_{\Omega^{\prime}}(X) \epsilon_{\Omega^{\prime}}^{2} d X$ is a Carleson measure with norm depending only on $C_{0}$ and on the Lipschitz character of $\Omega^{\prime}$.

Proof: It suffices to prove the lemma in the case where $\Omega=\mathbb{R}_{+}^{n+1}$. Let $\Omega$ denote the Lipschitz subdomain of $\mathbb{R}_{+}^{n+1}$ and fix a Carleson box $T(\Delta)$ associated to a surface ball $\Delta \subset \partial \Omega$. Let $X_{0}=\left(x_{0}, t_{0}\right)$ be the center of $T(\Delta)$. We consider two cases.

In case 1 , we assume that the diameter of $T(\Delta)$ is smaller than $\frac{1}{10} t_{0}$. Then if $(x, t) \in \mathbb{R}_{+}^{n+1}$ belongs to $T(\Delta), t_{0} \leq \frac{10}{9} t$, since $t_{0}=\left(t_{0}-t\right)+t \leq$ $\operatorname{diam} T(\Delta)+t$. Because $t \epsilon^{2}(x, t) d x d t$ is a Carleson measure, $|\nabla a(x, t)| \leq$ $\frac{C}{t}$, and hence $\epsilon_{\Omega}(x, t) \leq \frac{C}{t_{0}}$, by our assumption on $\operatorname{diam} T(\Delta)$. This gives

$$
\begin{aligned}
\int_{T(\Delta)} \delta_{\Omega}(X) \epsilon_{\Omega}^{2}(X) d X & \leq\left(\frac{C}{t_{0}}\right)^{2}[\operatorname{diam} T(\Delta)]^{n+2} \\
& \leq C(\operatorname{diam} T(\Delta))^{n} \\
& \leq C \sigma(\Delta),
\end{aligned}
$$

which is the Carleson condition on $\Omega$.

In case 2 , when $\operatorname{diam} T(\Delta)>\frac{1}{10} t_{0}$, we let $Q$ be the cube in $\mathbb{R}^{n}$ whose center $\left(x_{0}, 0\right)$ is the projection of $X_{0}$ and whose diameter is $(M+1) t_{0}$, where $M$ depends only on the Lipschitz character of $\Omega$ and is chosen so that $\operatorname{diam} T(\Delta) \leq M t_{0}$. With this choice, it is easy to verify that $T(\Delta) \subset T(Q)$. Thus, since $\delta_{\Omega}(X) \leq t$ and $\epsilon_{\Omega}(x, t) \leq \epsilon(x, t)$,

$$
\begin{aligned}
\int_{T(\Delta)} \delta_{\Omega}(X) \epsilon_{\Omega}^{2}(X) d X & \leq C \int_{T(Q)} t \epsilon^{2}(x, t) d x d t \\
& \leq C|Q| \\
& \leq C \sigma(\Delta) .
\end{aligned}
$$

Let now $L=\operatorname{div} a \nabla$ be an elliptic operator satisfying condition (2.7) of Theorem 2.6 defined in $\Omega$. We wish to establish the equivalence on 
subdomains $\mathcal{D}$ of $\Omega$, of $\|N(u)\|_{L^{p}(\partial \mathcal{D}, d \sigma)}$ and $\|S(u)\|_{L^{p}(\partial \mathcal{D}, d \sigma)}$ for solutions $L u=0$ in $\Omega$. Of the two inequalities, the domination of $\|N(u)\|_{L^{p}}$ by $\|S(u)\|_{L^{p}}$ is the more difficult to establish, and we deal with this first. We begin with a lemma which shows that the $L^{2}$ norm of a solution is bounded by $L^{2}$ norms of quantities involving $N$ and $S$. The idea (see 3.8 .1 of $[\mathbf{K K P T}]$ ) is to combine such inequalities on varying Lipschitz domains with a stopping time argument. We shall henceforth assume that $u$ is a solution to an operator $L=\operatorname{div} a \nabla$ whose coefficients satisfy conditions (2.7), and are also $\mathcal{C}^{\infty}$, and we shall obtain estimates which do not depend on the order of smoothness.

Lemma 3.2. Let $\Delta$ be the graph of a Lipschitz function $\phi(x)$ and let

$$
\mathcal{O}_{\phi}=\{(x, t):|x| \leq 1, \phi(x)<t<\phi(x)+1\} .
$$

Suppose that $L u=\operatorname{div} a \nabla u=0$ in $\mathcal{O}_{\phi}$, where a satisfies conditions 2.7. Then there exists a constant $C>0$ and a compact set $K \subset \mathcal{O}_{\phi}$, at distance $C$ from $\partial \mathcal{O}_{\phi}, C$ depending only on the Lipschitz constant of $\phi$ such that

$$
\begin{aligned}
& \int_{\Delta_{\frac{1}{2}}} u^{2} d \sigma \leq C \int_{\Delta_{\frac{3}{4}}} S_{1}^{2}(u) d \sigma \\
& +C\left(\int_{\Delta_{\frac{3}{4}}} S_{1}^{2}(u) d \sigma\right)^{\frac{1}{2}} \cdot\left(\int_{\Delta_{\frac{3}{4}}} N_{1}^{2}(u) d \sigma\right)^{\frac{1}{2}}+C \iint_{K} u^{2} d X .
\end{aligned}
$$

Remark. Recall that $N_{h}$ and $S_{h}$ denote nontangential maximal functions and square functions defined with respect to cones truncated at height $h \leq 1$, with aperture chosen so that they are compactly contained in $\mathcal{O}_{\phi}$. We will combine (3.3) with a stopping time argument, to obtain upper bounds for $N(u)$, as well as for $u$.

Proof: Set $\mathcal{O}=\mathcal{O}_{\phi}$. First, let $\psi(x, t)=\theta(x) \mu(t)$ be a product of $\mathcal{C}^{\infty}$ cut-off functions $\theta$ and $\mu$, supported in $\left\{|x| \leq \frac{3}{4}\right\} \times\left\{0 \leq t \leq \frac{3}{4}\right\}$. Our strategy is to use a special change of variables to map $\mathcal{O}$ onto a region in $\mathbb{R}_{+}^{n+1}$ and prove (3.3) by integration by parts. We will use $\psi(x, t)$ to localize our integrands. Thus we set $\eta_{t}(x)=t^{-n} \eta(x / t)$, where $\eta(\cdot)$ is an even $\mathcal{C}^{\infty}$ approximate identity supported in $\left\{|x| \leq \frac{1}{2}\right\}$, and set $\rho(x, t)=(x, c t+F(x, t))$ with $F(x, t)=\eta_{t} * \phi(x)$. For appropriate choice of $c$, depending on $\|\nabla \phi\|_{\infty}, \rho$ is a 1-1 map of $\mathbb{R}_{+}^{n+1}$ onto $\{(x, t): t>\phi(x)\}$ and this transformation gives rise to the Dahlberg-Kenig-Stein adapted distance function. 
Set $v(x, t)=u \circ \rho(x, t)$, where $u$ is a solution to $L u=0$. Then, in $\rho^{-1}(\mathcal{O}) \subset \mathbb{R}_{+}^{n+1}, v$ is a solution to $\tilde{L}=\operatorname{div} b \nabla$ where $b=\left(b_{i j}\right)$ satisfies the condition:

$$
t \beta^{2}(x, t) d x d t \text { is a Carleson measure }
$$

where

$$
\beta(x, t)=\sup \left\{\left|\nabla b_{i}(z, s)\right|: i, j=1, \ldots, n \text { and }(z, s) \in B_{\frac{t}{2}}(x, t)\right\} .
$$

Because the matrix $b$ is related to the original matrix by the formula

$$
b=|\operatorname{det} D \rho|\left(D\left(\rho^{-1}\right)\right)^{t} a \circ \rho(D \rho)^{-1},
$$

property 3.4 follows from the fact that $t|\nabla \nabla \rho(x, t)|^{2} d x d t$ is a Carleson measure.

Thus the quantity $\int_{\Delta_{\frac{1}{2}}} u^{2} d \sigma$ is bounded from above by

$$
\begin{aligned}
\int v^{2}(x, 0) \theta(x) \mu(0) d x= & -\int D_{t}\left(v^{2}(x, t) \psi(x, t)\right) d x d t \\
= & -\int 2 v(x, t) b_{n n}(x, t) D_{t} v(x, t) \frac{D_{t}(t)}{b_{n n}(x, t)} \psi(x, t) d x d t \\
& -\int v^{2}(x, t) D_{t} \psi(x, t) d x d t .
\end{aligned}
$$

The second summand above is bounded by the factor $\int_{K} u^{2}(X) d X$ where $K$ is compactly contained in $\mathcal{O}(K \Subset \mathcal{O})$, and in the first summand we have introduced the $(n, n)$ coefficient of the matrix $b$. This term is, after integration by parts, equal to

$$
\begin{aligned}
& \int 2 v(x, t) D_{t}\left(b_{n n}(x, t) D_{t}(v(x, t)) \frac{t}{b_{n n}(x, t)} \psi(x, t) d x d t\right. \\
+ & \int 2\left(D_{t} v(x, t)\right)^{2} \psi(x, t) t d x d t \\
+ & \int 2 v(x, t) D_{t} v(x, t) D_{t} \psi(x, t) t d x d t \\
& -\int 2 v(x, t) b_{n n}(x, t) D_{t} v(x, t) \psi(x, t) \frac{D_{t} b_{n n}(x, t)}{b_{n n}(x, t)^{2}} t d x d t \\
= & I+I I+I I I+I V .
\end{aligned}
$$


Term $I I$ is bounded by

$$
C \int_{(x, t) \in \mathcal{O} \cap\left\{|t| \leq \frac{3}{4}, \phi(x)<t<\phi(x)+1\right\}} \delta(X)|\nabla u(X)|^{2} d X \leq C \int_{\Delta_{\frac{3}{4}}} S_{1}^{2}(u) d \sigma
$$

where the subscripted $S_{1}$ denotes the square function with respect to cones truncated at height 1 . The constant $C$ depends on several parameters - the Lipschitz constant, the ellipticity constant of the matrix $a$, and the truncations of the cones - but not on the solution.

Term III is, by the Cauchy-Schwarz inequality, dominated by

$$
\begin{array}{r}
C\left(\int\left|D_{t} \psi(x, t)\right| v^{2}(x, t) d x d t\right)^{\frac{1}{2}} \cdot\left(\int t\left|D_{t} x(x, t)\right|^{2}\left|D_{t} \psi(x, t)\right| d x d t\right)^{\frac{1}{2}} \\
\leq C\left(\int_{K} u^{2} d X\right)^{\frac{1}{2}} \cdot\left(\int_{\Delta_{\frac{3}{4}}} S_{1}^{2}(u) d \sigma\right)^{\frac{1}{2}}
\end{array}
$$

For term $I V$, we will need the Carleson measure properties of $t\left|\nabla b_{n n}\right|^{2}$ as well as the upper and lower bounds on $b_{n n}(x, t)$ guaranteed by ellipticity:

$$
\begin{aligned}
& I V \leq C\left(\int v^{2}(x, t) \psi(x, t) t\left|\nabla b_{n n}(x, t)\right|^{2} d x d t\right)^{\frac{1}{2}} \\
& \cdot\left(\int t \psi(x, t)\left|D_{t} v(x, t)\right|^{2} d x d t\right)^{\frac{1}{2}} \leq\left(\int_{\Delta_{\frac{3}{4}}} N_{1}^{2}(u) d \sigma\right)^{\frac{1}{2}} \cdot\left(\int_{\Delta_{\frac{3}{4}}} S_{i}^{2}(u) d \sigma\right)^{\frac{1}{2}} .
\end{aligned}
$$

Only in the estimate on term $I$ do we use the equation that $v$ satisfies, obtaining

$$
\begin{aligned}
I & =-2 \int \sum_{(i, j) \neq(n, n)} D_{i}\left(b_{i j}(x, t) D_{j} v(x, t)\right) \frac{t v(x, t) \psi(x, t)}{b_{n n}(x, t)} d x d t \\
& =I_{1}+I_{2}
\end{aligned}
$$

where $I_{1}$ is the sum over $(i, j)=(n, j)$ with $j<n$ and $I_{2}$ is the sum over $(i, j)$ with $i<n$. 
In term $I_{2}$, we may integrate by parts with respect to $D x_{i}$ to obtain

$$
\begin{aligned}
I_{2}= & 2 \int \sum_{i<n} b_{i j} D_{j} v D_{i}\left\{\frac{v t \psi}{b_{n n}}\right\} d x d t \\
= & 2 \int b_{i j} D_{j} v D_{i} v \frac{t \psi}{b_{n n}} d x d t \\
& +2 \int \sum_{i<n} b_{i j} D_{j} v v t\left\{\frac{D_{i} \psi}{b_{n n}}-\frac{\psi D_{i} b_{n n}}{b_{n n}^{2}}\right\} d x d t \\
\leq & C \int|\nabla v|^{2} t \psi d x d t \\
& +C\left(\int|\nabla v|^{2} t|\nabla \psi| d x d t\right)^{\frac{1}{2}}\left(\iint v^{2} t|\nabla \psi| d x d t\right)^{\frac{1}{2}} \\
& +C\left(\int|\nabla v|^{2} t \psi d x d t\right)^{\frac{1}{2}}\left(\int v^{2} t \psi\left|b_{n n}\right|^{2} d x d t\right)^{\frac{1}{2}} \\
\leq & C \int_{\Delta_{\frac{3}{4}}} S_{1}^{2}(u) d \sigma+\left(\int_{\Delta_{\frac{3}{4}}} S_{1}^{2}(u) d \sigma\right)^{\frac{1}{2}} \cdot\left(\int_{K} u^{2} d \sigma\right)^{\frac{1}{2}} \\
& +\left(\int_{\Delta_{\frac{3}{4}}} S_{1}^{2}(u) d \sigma\right)^{\frac{1}{2}} \cdot\left(\int_{\Delta_{\frac{3}{4}}} N_{1}^{2}(u) d \sigma\right)^{\frac{1}{2}} \cdot
\end{aligned}
$$

We rewrite term $I_{1}$ as,

$$
\sum_{j<n} D_{n}\left(b_{n j} D_{j} v\right)=\sum_{j<n}\left(D_{n} b_{n j} \cdot D_{j} v+D_{j}\left(b_{n j} D_{n} v\right)-D_{j} b_{n j} \cdot D_{n} v\right) .
$$

When the derivatives fall on $b_{n j}$, we use the Carleson measure property of $\left|\nabla b_{i j}\right|^{2} t$ and for the summands $D_{j}\left(b_{n j} D_{j} v\right)$, with $j<n$, the situation is exactly that involved in the estimate of $I_{2}$. This completes the proof.

The fact that an inequality of the form (3.3) is valid above any Lipschitz graph can be used to generate a similar inequality with $\|N u\|$ replacing $\|u\|$, via a stopping time argument. To see this let us fix a Lipschitz function $\psi(x)$ and a solution $u$ defined in the region $\mathcal{O}_{\psi}$ of Lemma 3.2. Let $\Lambda$ denote the graph of $\psi(x)$, and fix an aperture $a$ for the cones used to define the nontangential maximal function $N u(x, \psi(x))$. The cones of aperture $8 a$ will be required to be compactly contained in $\mathcal{O}_{\psi}$. Then, the square function will be defined using cones of larger aperture than $a$, say $4 a$, for technical measures connected with proving good- $\lambda$ inequalities. All the cones at $(x, \psi(x))$, for 
$|x|<\frac{1}{4}$, will be at a height sufficient to insure that they are contained in $\left\{|x| \leq \frac{3}{4}, \psi(x) \leq \psi(x)+\frac{3}{4}\right\}$. We define the function $v(x, t)$ in all of $\{(x, t): t \geq \psi(x)\}$ by

$$
v(x, t)=u(x, t) \theta(x) \mu_{\alpha}(t-\psi(x))
$$

where $\theta \in C^{\infty}, 0 \leq \theta \leq 1, \theta(x)=1$ when $|x| \leq \frac{3}{4}, \operatorname{supp}(\theta) \subset\left\{|x|<\frac{7}{8}\right\}$ and $\mu_{\alpha}$ is also a $C^{\infty}$ cut-off function equal to 1 in $\left(0, \frac{\alpha}{2}\right)$ and supported in $(0, \alpha)$. Here $\alpha$ is a truncation parameter which may be taken to equal half the height of the truncated cones above. Define

$$
h_{j}(x)=\sup \left\{t \geq \psi(x): \sup _{(z, s) \in \Gamma_{a}(x, t)}|v(z, s)|>2^{j}\right\}
$$

where

$$
\Gamma_{a}=\{(z, s):|z-x| \leq a(s-t)\}
$$

is a cone with vertex at $(x, t)$.

The functions $h_{j}(x)$ were defined in $[\mathbf{K K P T}]$ and are inspired by a stopping time argument of $[\mathbf{F e}-\mathbf{S t}]$. We now prove that for any $v(x, t)$ such that $h_{j}(\cdot)<+\infty$, these $h_{j}$ are uniformly Lipschitz.

Lemma 3.5. The functions $h_{j}(x)$ are Lipschitz with constant $\frac{1}{a}$.

Proof: Fix $x_{1}$ and $x_{2}$ and suppose $h_{j}\left(x_{1}\right)=t_{1}$. If $\tilde{t}=t_{1}+\frac{1}{a}\left|x_{2}-x_{1}\right|$, then $\Gamma_{a}\left(x_{2}, \tilde{t}\right) \subset \Gamma_{a}\left(x_{1}, t_{1}\right)$ and so $\tilde{t} \geq \psi\left(x_{2}\right)$. Also, $h_{j}\left(x_{2}\right) \leq \tilde{t}$, for if not, there would exist a cone $\Gamma_{a}\left(x_{2}, t_{2}\right)$, properly contained in $\bar{\Gamma}_{a}\left(x_{1}, t_{1}\right)$, containing a point $(z, s)$ for which $|v(z, s)|>2^{j}$. But this would imply that $h_{j}\left(x_{1}\right)>t_{1}$, a contradiction. Now let $\tilde{\tilde{t}}=t_{1}-\frac{1}{a}\left|x_{2}-x_{1}\right|$. If $\tilde{\tilde{t}} \geq \psi\left(x_{2}\right)$, then a similar argument as above gives $h_{j}\left(x_{2}\right) \geq \tilde{\tilde{t}}$. Therefore, $\left|h_{j}\left(x_{1}\right)-h_{j}\left(x_{2}\right)\right| \leq \frac{1}{a}\left|x_{2}-x_{1}\right|$. If $\tilde{\tilde{t}}<\psi\left(x_{2}\right)$, then in any case

$$
\begin{aligned}
\left|h_{j}\left(x_{2}\right)-h_{j}\left(x_{1}\right)\right| & \leq \max \left\{\left|t_{1}-\tilde{t}\right|,\left|t_{1}-\psi\left(x_{2}\right)\right|\right\} \\
& \leq \frac{1}{a}\left|x_{2}-x_{1}\right| .
\end{aligned}
$$

Lemma 3.6. Let $\Gamma_{a}^{\beta}(x, \psi(x))$ denote a cone with vertex $(x, \psi(x))$, aperture $a$, truncated at height $\beta$. Choose $\beta$ so that $\Gamma_{4 a}^{\beta}(x, \psi(x)) \subset\{|x| \leq$ $\left.\frac{3}{4}, \psi(x) \leq t \leq \psi(x)+\frac{3}{4}\right\}$ when $|x| \leq \frac{1}{4}$. Let $N_{a, \beta / 2} u$ and $S_{4 a, \beta} u$ denote, respectively, the nontangential maximal function and the square function defined using the cones $\Gamma_{a}^{\beta / 2}$ and $\Gamma_{4 a}^{\beta}$. Let

$E_{j, \rho}=\left\{|x| \leq \frac{1}{4}\right\} \cap\left\{x: N_{a, \beta / 2} u(x, \psi(x))>2^{j}, S_{4 a, \beta} u(x, \psi(x)) \leq \rho 2^{j}\right\}$. 
Then, there exists a choice of $\rho$ so that for all $x \in E_{j, \rho}$, there is a cube $J$ with $x \in J$ and $J \subset\left\{|x| \leq \frac{3}{4}\right\}$ and for which $\left|u\left(z, h_{j}(z)\right)\right|>2^{j-1}$ for all $z \in J$.

Proof: The proof of this fact is given in (3.14) of [KKPT].

We shall also require a rescaled version of Lemmas 3.2 and 3.6, which we record as follows.

Let $\mathcal{O}_{r}=\{(x, t):|x| \leq 2 r, \psi(x)<t<\psi(x)+2 r\}$ and suppose $L u=0$ in $\mathcal{O}_{r}$. Then, if $A_{r}$ is any point of $\mathcal{O}_{r}$ whose distance to $\partial \mathcal{O}_{r}$ is approximately $r$, there exists a $C=C\left(\left\|\psi^{\prime}\right\|_{\infty}\right)$ such that

$$
\begin{aligned}
& \int_{\Delta_{\frac{r}{2}}} u^{2} d \sigma \leq C \int_{\Delta_{\frac{3}{4} r}} S_{r}^{2}(u) d \sigma \\
& \quad+C\left(\int_{\Delta_{\frac{3}{4} r}} S_{r}^{2}(u) d \sigma\right)^{\frac{1}{2}} \cdot\left(\int_{\Delta \frac{3}{4} r} N_{r}^{2}(u) d \sigma\right)^{\frac{1}{2}}+C r u^{2}\left(A_{r}\right) .
\end{aligned}
$$

The inequality above is exactly a rescaled version of (3.3) with the term $\iint_{K_{r}} u^{2} d X$ replaced by $r u^{2}\left(A_{r}\right)+\int_{\Delta \frac{3}{4} r} S_{r}^{2}(u) d \sigma$.

To rescale Lemma 3.6, observe that if $\Gamma_{4 a}^{\beta^{\prime}}\left(x, \psi(x) \subset\left\{|x| \leq \frac{3}{4} r, \psi(x) \leq\right.\right.$ $\left.t \leq \psi(x)+\frac{3}{4} r\right\}$ when $|x| \leq \frac{1}{4} 4$, and $\beta^{\prime}=\beta r$, then the choice of $\rho$ is independent of $r$.

Theorem 3.8. Let $\Omega \subset \mathbb{R}^{n}$ be a bounded Lipschitz domain, with Lipschitz character $\left(M, N, C_{0}\right)$, and let $u$ be a solution to $\operatorname{div} a \nabla u=0$ in $\Omega$, as in (2.6). Suppose $u\left(P^{*}\right)=0$ for some $P^{*} \in \Omega$. Let $d \sigma$ denote surface measure on $\partial \Omega$. Assume, for $p>2$, that $A=\left\|S_{4 a}(u)\right\|_{L^{p}(\partial \Omega, d \sigma)}<+\infty$. Then, given $\gamma<1$, there exists a constant $C(\gamma)$, tending to zero as $\gamma \rightarrow 0$ such that for all $\lambda>C A$,

$$
\begin{array}{r}
\sigma\left\{N_{\alpha}(u)>2 \lambda, M\left(S_{4 a}(u) \leq \gamma \lambda\right), M\left(S_{4 a}^{2}(u)\right)^{\frac{1}{4}} \cdot M\left(N_{a}^{2}(u)\right)^{\frac{1}{4}} \leq \gamma \lambda\right\} \\
\leq C(\gamma) \sigma\left\{N_{a}(u)>\frac{\lambda}{32}\right\},
\end{array}
$$

where

$$
M f(Q)=\sup \left\{\int_{\Delta} f(P) \frac{d \sigma(P)}{\sigma(\Delta)}: \Delta \subset \partial \Omega, \Delta \ni Q\right\} .
$$

Corollary 3.9. $\left\|N_{a}(u)\right\|_{L^{p}(d \sigma)} \leq C\left\|S_{4 a}(u)\right\|_{L^{p}(d \sigma)}$, for $a, \Omega$, $\sigma$, and $u$ as in Theorem 3.8, for all $p>2$.

The proof of the corollary is standard from the good- $\lambda$ inequality. The proof of Theorem 3.8 differs from the proof of application (3.1) 
in $[\mathbf{K K P T}]$. There, it was necessary to prove the $L^{p}$-inequalities on graphs, so as to use the G. David arguments building on the case of small Lipschitz constant. The need to 'build up' from the small constant case makes the arguments much more technical, requiring a more elaborate localization scheme.

Proof of Theorem 3.8: (We omit those details which are standard, and can be found in many references.) Let $\left\{\Delta_{l}\left(Q_{l}, r_{l}\right)\right\}$ be a Whitney decomposition of $\left\{N_{a}(u)>\frac{\lambda}{32}\right\}$. That is, $\left\{N_{a}(u)>\frac{\lambda}{32}\right\} \subset \cup_{l} \Delta_{l}, \Delta_{l}=$ $B_{l}\left(Q_{l}, r_{l}\right) \cap \partial \Omega$ with $\sum_{l} \chi_{B_{l}}(X) \leq C(M)$, and each $8^{n} \Delta_{l}=B_{l}\left(Q_{l}, 8 r_{l}\right) \cap$ $\partial \Omega$ is contained in the graph of a Lipschitz function. Moreover, there exists a parameter $r_{0}=r_{0}\left(M, N, C_{0}\right)$ such that if $r_{l} \leq r_{0}$, then $B\left(Q_{l}, 2^{n} r_{l}\right) \cap$ $\partial \Omega$ contains a point $Q_{l}^{*}$ where $N_{a}(u)\left(Q_{l}^{*}\right) \leq \frac{\lambda}{32}$.

Since we make the a priori assumption that $\left\|S_{4 a}(u)\right\|_{L^{p}}<+\infty$, interior estimates together with the normalization on $u$ handles the estimates on the 'large' Whitney balls - those with radius larger that the parameter $r_{0}$ (see [D3]). Thus we fix a $\Delta=\Delta_{l}$ with $r_{l} \leq r_{0}$ in the Whitney decomposition and let

$$
\begin{aligned}
F=\Delta \cap\left\{N_{a}(u)>2 \lambda, M\left(S_{4 a}(u)\right)\right. & \leq \lambda \gamma, \\
& \left.M\left(S_{4 a}^{2}(u)\right)^{\frac{1}{4}} \cdot M\left(N_{a}^{2}(u)\right)^{\frac{1}{4}} \leq \gamma \lambda\right\} .
\end{aligned}
$$

By choosing $\gamma$ sufficiently small, we can ensure that $N_{a, \beta_{l}}(u)>\frac{\lambda}{2}$ in $F$ where $\beta_{l}$ is a truncation of the cones which equals $c_{1} r_{l}$, where $r_{l}$ is the radius of $\Delta_{l}$, and which satisfies the conditions of Lemma 3.6, rescaled to $r_{l}$.

Now set $\lambda=2^{j}$, and define the Lipschitz function $h_{j}(x)$ as in Lemma 3.5 relative to the Lipschitz function $\phi$, where $8 \Delta_{l}$ is contained in the graph of $\phi$. The function $v(x, t)$ in the definition of $h_{j}$ will be supported in $T\left(2 \Delta_{l}\right)$ since the cut-off functions are tailored to scale $r_{l}$. Let $\Delta_{l}^{*}=$ $\left\{\left(x, h_{j}(x)\right):(x, \phi(x)) \in 4 \Delta_{l}\right\}$. Then by 3.6, for $\gamma<\rho$, for all $(x, \phi(x)) \in$ $F$, we have $M_{j}\left(u \chi_{\Delta_{l}^{*}}\right)\left(x, h_{j}(x)\right)>\frac{2^{j}}{16}$, where $M_{j}$ denotes the HardyLittlewood maximal function on the graph $h_{j}$ :

$$
M_{j} F\left(x, h_{j}(x)\right)=\sup \left\{\int_{I} F\left(z, h_{j}(z)\right) d \sigma_{j}(z): x \in I\right\} .
$$

Let $A_{r_{l}}$ be a point in $\mathcal{O}_{l}$ satisfying inequality 3.7 . Since $\Delta_{l}$ is one of the 'small' Whitney balls, the existence of the point $Q_{l}^{*}$ where $N_{a}(u)\left(Q_{l}^{*}\right) \leq$ $\frac{2^{j}}{32}$ means that

$$
M_{j}\left(\tilde{u} X_{\Delta_{l}^{*}}\right)\left(x, h_{j}(x)\right)>\frac{2^{j}}{32} \text { if } \tilde{u}(X)=u(X)-u\left(A_{l}\right) .
$$


Thus, by replacing $u$ by $\tilde{u}$, we may assume that $u$ vanishes at $A_{l}$. (This means that the constant $\gamma$ in the definition of $F$ should be replaced by $\sqrt{\gamma}$.) Therefore, by the weak type inequality for the maximal function,

$$
\sigma(F) \leq C 2^{-2 j} \int_{\Delta_{l}^{*}} u^{2}\left(x, h_{j}(x)\right) d \sigma .
$$

We now apply inequality (3.7) to bound the above expression by two terms, one of which is

$$
C 2^{-2 j} \int_{2 \Delta_{l}^{*}} S_{4 a, 2 \beta_{l}, h_{j}}^{2}(u) d \sigma_{j}
$$

The indices on the square function show that this is defined with respect to the graph $h_{j}(x)$ using truncated cones $\left(2 \beta_{l}\right)$ of aperture $a$.

The cones $\Gamma_{4 a, 2 \beta_{l}, h_{j}}\left(x, h_{j}(x)\right)$ are always contained in the cones $\Gamma_{4 a}(x, \phi(x))$ so, in dimension $n=2$, the square functions

$S_{4 a, 2 \beta_{l}, h_{j}} u\left(x, h_{j}(x)\right)$ are dominated pointwise by the square functions $S_{4 a}(u)(x, \phi(x))$. Then the quantity (3.10) would be bounded by $M\left(S_{4 a}(u)(x, \phi(x)) \cdot \sigma\left(8 \Delta_{l}\right)\right.$, for $(x, \phi(x)) \in F$, which is, in turn, bounded by $\left(\gamma 2^{j}\right)^{2} \sigma\left(8 \Delta_{l}\right)$. In dimension $n>2$, the desired upper bound can be obtained by carrying out the integration:

$$
\int_{2 \Delta_{l}^{*}} S_{4 a, 2 \beta_{l}, h_{j}}^{2} u d \sigma_{j} \lesssim \iint_{T\left(3 \Delta_{l}\right)} \delta_{j}(X)|\nabla u(X)|^{2} d X
$$

where $\delta_{j}(X)=\operatorname{dist}\left(X\right.$, graph of $\left.h_{j}\right)$. Then, since $\delta_{j}(X) \leq \delta(X)$, we recover $\int_{4 \Delta_{l}} S_{4 a}^{2}(u)(Q) d \sigma(Q)$, and the upper bound $\left(\gamma 2^{j}\right)^{2} \sigma\left(\Delta_{l}\right)$.

The other term arising from 3.7 in the bound for 3.10 is handled similarly. Summing our estimates on $l$ proves 3.8 .

The arguments for the converse inequality to that of 3.8 ,

$$
\left\|S_{a}(u)\right\|_{L^{p}(d \sigma)} \leq C\left\|N_{4 a}(u)\right\|_{p(d \sigma)}
$$

are similar, but simpler. Here we do not need the stopping time argument, nor the introduction of the $h_{j}$ 's. We omit the details.

We also note that parabolic analogs of Theorem 2.6 hold, with similar proofs. The same applies to Theorem 2.8 if the Carleson measure norm in (2.9) is sufficiently small, for then the parabolic measure is a doubling measure $([\mathbf{H L}])$. Whether or not doubling is true for parabolic measures when the norm in (2.9) is large remains an interesting open problem. 


\section{Bibliography}

[CFK] L. A. Caffarelli, E. B. Fabes and C. E. Kenig, Completely singular elliptic-harmonic measures, Indiana Univ. Math. J. 30(6) (1981), 917-924.

[CFMS] L. A. Caffarelli, E. B. Fabes, S. Mortola and S. Salsa, Boundary behavior of nonnegative solutions of elliptic operators in divergence form, Indiana Univ. Math. J. 30(4) (1981), 621-640.

[CF] R. R. Coifman and C. Fefferman, Weighted norm inequalities for maximal functions and singular integrals, Studia Math. 51 (1974), 241-250.

[D1] B. E. J. DAhlBerG, On the Poisson integral for Lipschitz and $C^{1}$-domains, Studia Math. 66(1) (1979), 13-24.

[D2] B. E. J. DAHLBERG, Poisson semigroups and singular integrals, Proc. Amer. Math. Soc. 97(1) (1986), 41-48.

[D3] B. E. J. DAhlbERG, Weighted norm inequalities for the Lusin area integral and the nontangential maximal functions for functions harmonic in a Lipschitz domain, Studia Math. 67(3) (1980), 297-314.

[FJK] E. B. Fabes, D. S. Jerison and C. E. Kenig, Multilinear Littlewood-Paley estimates with applications to partial differential equations, Proc. Nat. Acad. Sci. U.S.A. 79(18) (1982), $5746-5750$.

[Fe-St] C. Fefferman and E. M. Stein, $H^{p}$ spaces of several variables, Acta Math. 129(3-4) (1972), 137-193.

[FKP] R. A. Fefferman, C. E. Kenig and J. Pipher, The theory of weights and the Dirichlet problem for elliptic equations, Ann. of Math. (2) 134(1) (1991), 65-124.

[GT] D. Gilbarg and N. S. Trudinger, "Elliptic partial differential equations of second order", Grundlehren der Mathematischen Wissenschaften [Fundamental Principles of Mathematical Sciences] 224, Springer-Verlag, Berlin-New York, 1983.

[HL] S. Hofmann and J. Lewis, The Dirichlet problem for parabolic operators with singular drift terms, to appear in Trans. Amer. Math. Soc.

[JK] D. S. Jerison and C. E. Kenig, The Dirichlet problem in nonsmooth domains, Ann. of Math. (2) 113(2) (1981), $367-382$. 
[KKPT] C. Kenig, H. Koch, J. Pipher And T. Toro, A new approach to absolute continuity of elliptic measure, with applications to non-symmetric equations, Adv. Math. 153(2) (2000), 231-298.

[M] B. Muckenhoupt, Weighted norm inequalities for the Hardy maximal function, Trans. Amer. Math. Soc. 165 (1972), $207-226$.

Department of Mathematics

Brown University

Box 1917

Providence, Rhode Island 02912

U.S.A.

E-mail address: CKenig@math.uchicago.edu

E-mail address: Jill_Pipher@Brown.edu

Rebut el 6 de juny de 2000. 